\title{
HL7 balloting process for the Implementation Guide for Syndromic Surveillance
}

\author{
Peter Hicks $^{* 1}$, Emilie Lamb ${ }^{2}$, Shandy Dearth ${ }^{2}$ and Dave Trepanier ${ }^{2}$
}

${ }^{1} \mathrm{CDC}$, Atlanta, GA, USA; ${ }^{2}$ SDS, Boston, MA, USA

\section{Objective}

To provide a forum to engage key stakeholders to discuss the process for updating and revising the Implementation Guide (IG) for Syndromic Surveillance (formerly the PHIN Message Guide for Syndromic Surveillance) and underscore the critically of community and stakeholder involvement as the Implementation Guide is vetted through the formal Health Level Seven (HL7) balloting process in 2018.

\section{Introduction}

Syndromic surveillance seeks to systematically leverage health-related data in near "real-time" to understand the health of communities at the local, state, and federal level. The product of this process provides statistical insight on disease trends and healthcare utilization behaviors at the community level which can be used to support essential surveillance functions in governmental public health authorities (PHAs). Syndromic surveillance is particularly useful in supporting public health situational awareness, emergency response management, and outbreak recognition and characterization. Patient encounter data from healthcare settings are a critical inputs for syndromic surveillance; such clinical data provided by hospitals and urgent care centers to PHAs are authorized applicable local and state laws. The capture, transformation, and messaging of these data in a standardized and systematic manner is critical to this entire enterprise.

In August 2015, a collaborative effort was initiated between the CDC, ISDS, the Syndromic Surveillance Community, ONC and NIST to update the national electronic messaging standard which enables disparate healthcare systems to capture, structure, and transmit administrative and clinical data for public health surveillance and response. The PHIN Messaging Guide for Syndromic Surveillance -Release 2.0 (2015) provided an HL7 messaging and content reference standard for national, syndromic surveillance electronic health record technology certification as well as a basis for local and state syndromic surveillance messaging implementation guides. This standard was further amended with the release of the PHIN Messaging Guide for Syndromic Surveillance - Release 2.0, Erratum (2015) and the HL7 Version 2.5.1 PHIN Messaging Guide for Syndromic SurveillanceRelease 2.0, NIST Clarifications and Validation Guidelines, Version 1.5 (2016). ISDS is now engaged in a process, supported by a CDC Cooperative Agreement, to formally revise the existing guide and generate an HL7 V 2.5.1 Implementation Guide (IG) for Syndromic Surveillance v2.5 for HL7 balloting in 2018.

This roundtable will provide a forum to present and discuss the HL7 Balloting process and the outstanding activities in which the Syndromic Surveillance community must participate during the coming months for this activity to be successful.

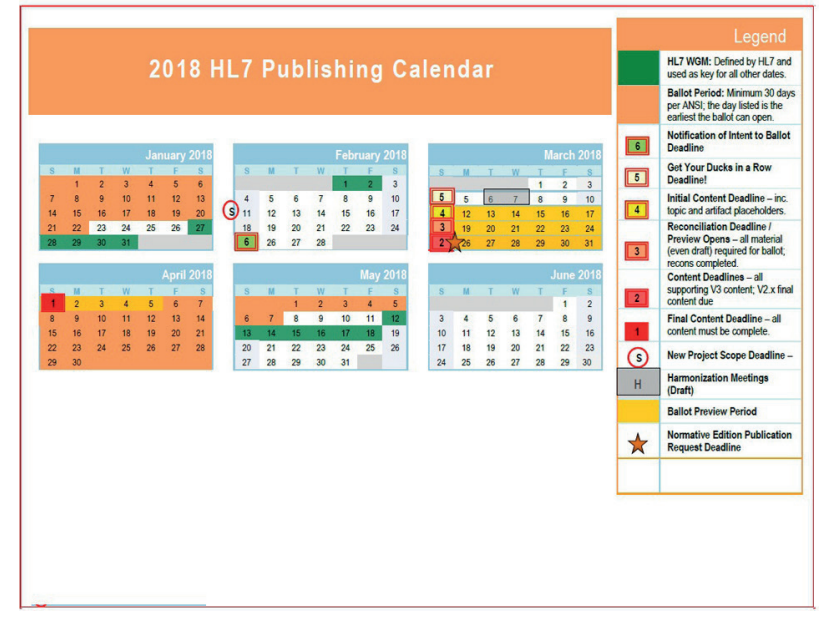

Keywords

Messaging Guide; Syndromic Surveillance; HL7

\section{*Peter Hicks}

E-mail: phicks@cdc.gov 